\title{
Perceived Social Support and Life Satisfaction: A Mediating Role of Quality of Life
}

\author{
Dr. Preeti Tarkara \\ ${ }^{a}$ Assistant Professor, Institute of Business Management, GLA University, Mathura, India. \\ E-mail: preeti.tarkar@gla.ac.in
}

Article History: Received: 11 January 2021; Accepted: 27 February 2021; Published online: 5 April 2021

\begin{abstract}
The purpose of the study is to examine the association between perceived social support, quality of life and life satisfaction after retirement through empirical examination. A sample of 300 government retired professionals was selected for conducting the study. Snowball sampling technique was used to collect the data for the study. In order to collect the data, a questionnaire was administered. SEM through AMOS was applied for data analysis to test the hypothesis. Based on the findings that quality of life of retired persons is significantly affected by the social support. Further, results show that life satisfaction is significantly affected by the quality of life. Overall model is significant. In this research the relationships between life satisfaction, quality of life and perceived social support were established. In order to enhance the quality of life and life satisfaction, family and friends support plays an important role in old age.
\end{abstract}

Keywords: Family Support, Friends Support, Life Satisfaction, Quality of Life, Structural Equation Modeling.

\section{Introduction}

Retirement is a stage of life when people get public support from the social security system and get an old age pension for the survival because retiree does not perform any action to gain income. Retirement pension and former savings are the primary sources for their survival.

The main problems which older people face are the lack of economic resources, health problems, lack of emotional support and idleness after retirement. This state of affairs becomes a social problem as a considerable number of people feel it as a problem. Growing age is not a problem, but many other problems are associated with this make it is a problem. These problems are related to society, culture, economic and political system. Problems of the elderly are more or less similar all over the world.

In the aging process, there are several transitions. Retirement is considered as a most influential event to affect older peoples' quality of life among these transitions. Retirement is generally considered as a state in which a person withdraws from working life.

The social status of a retired person changes after retirement, as before retirement, he was treated as a worker and after retirement, he is treated as retired which affects all domains of life. Retirement is also responsible for the transformation of an individual's physical and social world and also responsible for the overall life change because people leave the career job. The quality of life, ability and the perception of a retiree are affected by this transformation of life. A retired person often faces a plethora of problems because of the absence of secure and adequate income to support themselves for their basic needs. A few specific problems (Aging \& Retirement Issues, 2017) related to the retired persons after retirement are mentioned below:

Transitioning to retired life: it is assumed by some retired persons that retirement is a difficult stage of life in which people face adjustment problems and perceive aimless life. On the other hand, retirement is a most awaited moment of life, and resulting change can affect the mental and physical health. It is difficult for the retired person to face the situations after retirement because no longer s/he is useful or productive. It has become essential for the retirees to search the ways or occupy those roles in which s/he can feel useful again.

Dealing with loneliness: At this stage of life, most of the children get involved in their own families. Thus, this movement of children for settling their families and retirement happens together. It is difficult for every person to live life alone, but it is more difficult for retired persons. After retirement, the retiree has sufficient time to spend with family and friends, but most of the time retiree has to live alone without the family which results in social isolation which can lead to depression. Finally, it encourages the old age persons to withdraw, and it is detrimental for the emotional, mental and physical well-being.

Thus, the research paper aims to find out the relationship between perceived social support, life satisfaction and quality of life after retirement through empirical examination. 


\section{Conceptual Framework and Hypotheses Development}

\subsection{Perceived Social Support}

Pilisuk and Parks (1983) defined social support as "a set or range of regular interpersonal transactions that assist the individual in meeting physical, psychological and social needs."

The need for social support was highlighted by Biegel, Shore, Gordon (1984). They said that "the elderly have a greater need for social support than other population groups. One of the major issues for the elderly that creates a need for intervention is the continuum of loss of experience in body functioning, sensory functioning, mental functioning, family and peer group supports, income, self-image, self-esteem, control and power."

This type of social support indicates the extent to which interpersonal relationships between members of a social group fulfill essential functions. Researchers have stressed more on the structural aspects than the functional aspects, and the psychological aspects including perceived quality of support remain severely overlooked.

Shanas (1979) suggested that family becomes an essential source of social support during illness. His study was based on elderly persons aged 65 years and older. After the retirement, the social support from the children, peers and friends are reported by the older adults. The employment and retirement decisions are related to the family. Life course perspective highlights that a valuable sphere of life that interacts with the sphere of employment status is family (Szinovacz, 2003).

\subsection{Quality of Life}

Farquhar (1995) defined quality of life as "is a problematic concept as different people value different things" (p. 1440). This is very true that every person or even the same person may give importance to different things, depending upon the health, wealth and according to a certain period of life.

Quality of life is defined by different persons by considering the different aspects of life. Andrews \& Withey (1976) defined the QoL as an affective response of a person to the values and role situation. This definition clearly states that QoL depends on the individual's reaction to the circumstances, his/her values and role.

Most of the previous studies defined the QoL in terms of health or medical. But now it has been clear that QoL also considered various other factors. It is also affected by other areas such as social policy, social work, environmental study, psychology and sociology.

Further, according to Bowling and Windsor (2001), there are two perspectives to define the QoL that are the micro and macro term. The micro term includes the individual and subjective terms and macro term includes the objective and societal term. WHO QoL Group's definition is the sum of all the individual nature of QoL (WHOQOL, 1995):

"an individual's perception of their position in life in the context of the culture and value systems in which they live and in relation to their goals, expectations, and standards and concerns. It is a broad-ranging concept affected in a complex way by the persons' physical health, psychological state, level of independence, social relationships, and their relationships to salient features of environment".

WHO defines Quality of Life as "individuals' perception of their position in life in the context of the culture and value systems in which they live and in relation to their goals, expectations, standards and concerns." According to this definition, the individual's perception of QoL is closely related to a person's living standard, expectations and concerns about life.

Personal beliefs, social relationships, level of independence, psychological state, person's physical health and the relationship between these factors and environment affect the quality of life in a complex way. All these factors are important for the individuals as well. If we consider age as an important factor that affects the QoL then some additional factors related to aging are also considered.

McCall (1975) defined the quality of life concept in terms of happiness, as to approach the quality of life measurement the best method is to how much 'happiness requirements' of people are fufilled and requirements are necessary for an individual to achieve the happiness but not sufficient to anyone. Often, quality of life is 
measured in objective quality of life and subjective quality of life and these measures (objective and subjective quality of life) can be defined as, the fulfillment of the cultural and societal demands of a person for physical well-being, social status and material wealth is known as objective quality of life. The subjective aspect of quality of life is defined as the being satisfied and good feeling, in general, is known as subjective quality of life. Most of the time, Quality of life is defined as the subjective well-being and it is very important to understand the quality of life to understand the subjectivity of the concept.

Quality of life can be understood in terms of the important possibilities for a person and QOL depends on the degree to which a person enjoys these in his/her life. Every individual has some limitations and opportunities in his/her life and attainment of these possibilities reflect the interaction of environment and personal factors.

Thus, Quality of life (QOL) can be understood as the good life which means living a high-quality life. A number of quality of life theories have proved this notion about the quality of life as it can be observed from the objective and subjective dimensions of well-being. The combination of the objective and subjective aspects of quality of life is known as integrative quality of life theory. IQOL theory incorporates the following aspects such as objective aspects, fulfilment of the needs, realizing life potential, the biological information system ("balance"), happiness, meaning in life, satisfaction with life and well-being. The integrative theory of the quality of life derived the questions to the global quality of life.

\subsubsection{Family Support and Quality of Life}

Previous studies (Lena, Kumar, Padma, Kamath \& Kamath, 2009; Okumagba, 2011; Kaur, Kaur \& Venkateashan, 2015; Yuh \& Choi, 2017) showed the relationship between the dimensions of quality of life and social support. After retirement, retired people spend their free time at home in various leisure activities. They spend time in playing with their grandchildren, chatting with friends, relax in the sunlight, performing some household duties for instance: cleaning, cooking, gardening, watching TV, worshipping, knitting, reading the newspaper and rearranging the house. However, at a broad level, retirement is considered a family affair. Retirement also affects the quality of life of old age person, and there is a relationship between family support and quality of life. It has been confirmed that older people who experienced a good quality of life has a great family support. Naing, Nanthamongkolchai and Musawaengsub (2010) confirmed that there is a significant relationship between quality of life old age person and family relationship. Kaur et al. (2015) conducted a similar study on the family support and quality of life, and they find out that family support is an essential factor which affects the quality of life of a person in old age. Yuh and Choi (2017) find out that the quality of life is enhanced by family support. Thus, to examine the association between family support and quality of life, the following hypothesis is proposed.

\section{$\mathrm{H}_{1 \mathrm{a}}$ :There is a significant association between Family Support and Quality of Life.}

\subsubsection{Friends Support and Quality of Life}

Friends support is a great moral support for an older adult. Rawlins (2017) mentioned in his book that friendship matters a lot in old age. friends satisfies the social support for the older adults. Unsar, Erol and Sut (2016) stated in their study that friends support has a significant impact on the quality of life of the older adults. Thus, the relationship between friends support and quality of life examined by using the following hypothesis:

$\mathrm{H}_{1 \mathrm{~b}}$ : There is a significant association between Family Support and Quality of Life.

\subsection{Life Satisfaction}

According to Berg et al. (2009), life satisfaction has been a major concern. It is a holistic view, encompassing the overall lifespan of an individual and is not a specific aspect. Chadha and Van Willigen, (1995) stated that in relation to psychological well-being, the attitudes of the individuals about their past, present as well as future is considered as the life satisfaction. When the aging couple is free from the responsibilities of children, there is more scope for sharing among couples and strengthens intimacy (Jamuna \& Ramamurthi, 1988). Greater satisfaction with the present life is caused by the favourable and positive perception of the relationship with spouse (Ramamurthi \& Jamuna, 1991). Further, it was observed that satisfying relationship with children, peers and others contribute to satisfaction with present life (Ramamurti \& Jamuna, 1993). In the same study, the researchers opined that happy ageing is "characterized by satisfaction with one's present life and a psychological state of happiness and content with the existing condition." Results from the study showed that a satisfying relationship with spouse adds to happiness in later years. 


\subsubsection{Quality of Life and Life Satisfaction}

Shin (1979) observed that there are numerous factors which influence the quality of life. However, the definition of quality of life is given as the person's satisfaction with the dimensions of the ideal or pursued quality of life. It can be assessed by the cultural environment and the value system of a person (Gilgeous, 1998; Suber, 1996; Fitzpatrick, 1996). The concept of quality of life is also affected by the external circumstances. A slight change in life values can be determined by the living conditions, and it can also affect the measurement and understanding of the quality of life. There are many conditions that decide the quality of life. These factors may be social relationships and social support, independence level, health status and prospects of health care, stress and crisis, spiritual, physical, dwelling, ecological factors, personal and family life, employment, moral attitude, material well-being and income, etc. (Phillips, 2006).

Many a time, to evaluate the quality of life, spillover theory can be applied. In one sphere, spillover theory maintains the satisfaction of a person and the other spheres of quality of life influence the satisfaction level. Human consciousness has a particular hierarchy of life spheres. A higher level of satisfaction in one life sphere enhances the level of satisfaction in other spheres which means the satisfaction with the full quality of life is increased by the high quality of work life (spillover rises from bottom to top). Though, dissatisfaction from the one sphere of life does not have significant influence on the satisfaction. For instance, according to Furmonavicius (2003), if a person is not satisfied with one sphere of life such as s/he is not satisfied from the job than s/he pays more attention to the experience and family and gets a maximum satisfaction from the family that increases the quality of family life satisfaction. Thus, the following hypothesis is proposed.

$\mathrm{H}_{2}$ : There is a significant effect of Quality of Life on Life Satisfaction.

$\mathrm{H}_{3 \mathrm{a}}$ :There is a direct significant effect of family support on Life Satisfaction.

$\mathrm{H}_{3 a}$ :There is a direct significant effect of friends support on Life Satisfaction.

\section{Research Methodology}

The study was empirical in nature. The data was collected from government retired persons. In order to collect the data, a well administered questionnaire was used. The data was collected by using the snowball sampling method. Referrals were sought to collect the data. 360 responses were collected for data analysis. 300 questionnaires were usable from them. Therefore, 60 questionnaires were rejected and not considered for further analysis. Response rate was more than 80 (83.3) per cent.

Age of the respondents lies between 60 to 75 years. The average age of the respondents is 66 years ( $\mathrm{SD}=$ 5.19; range: 60-75). On the basis of gender classification, 94 per cent male $(n=282)$ and female was only 6 per cent $(\mathrm{n}=18)$.

\subsection{Measures}

\subsubsection{Perceived Social Support}

In order to measure family support and friends support eight items were adapted from MSPSS. This multidimensional scale was developed in 1988 by Zimet et al.

\subsubsection{Quality of Life}

Quality of life was measured through the Quality of Life Scale developed by Cummins (1997). This scale has been designed in such a manner that it can be applied to all populations for measuring the quality of life.

\subsubsection{Life Satisfaction}

In order to measure Life Satisfaction, I used Satisfaction with Life Scale developed by Diener, Emmons, and Larson (1985). For measuring the life satisfaction five items are given in this scale. Five point rating scale strongly disagree (1) to strongly agree (5) was used.

\subsection{Reliability Analysis}

Table 1. Constructs and Their Reliabilities 
Table 2. Direct

Effects of the

\begin{tabular}{|l|l|l|l|}
\hline Hypothesized path & Direct effect & Indirect effect & Total effect \\
\hline Fam QOL $\longrightarrow$ & .279 & - & .279 \\
Fri QOL $\longrightarrow$ & .264 & - & .164 \\
\hline QOL LS $\longrightarrow$ & .763 & - & .763 \\
\hline Fam LS $\longrightarrow$ & - & .213 & .213 \\
Fri LS $\longrightarrow$ & - & .202 & .202 \\
\hline \multicolumn{4}{|c}{ Variables on the Dependent Variables } \\
\hline Construct & No. of Items Cronbach's Alpha \\
\hline Family Support & 4 & .815 \\
Friends Support & 4 & .848 \\
Quality of Life & 6 & .813 \\
\hline Life Satisfaction & 5 & .758 \\
\hline
\end{tabular}

and Indirect

Independent

In order to measure the reliability of the scale, cronbach's alpha is used. The above table shows that the values of cronbach's alpha are greater than 0.7. Thus, all constructs are reliable. In table 1 the values of all constructs are greater than 0.7 .

\section{Data Analysis and Interpretation}

Structural equation modeling (SEM) by using AMOS is used to analyze the data. It is a confirmatory factor analysis (CFA). SEM is a mixture of multiple regression and factor analysis and it is used to analyze the relationship between independent and dependent variables. In which life satisfaction is used as a dependent variable, quality of life used as a mediating variable, family support and friend support both used as the independent variables. Figure 2 shows the relationship between the independent and dependent variables.

From the above table, it is clear that quality of life has largest positive effect on the life satisfaction. family support and friends support have direct positive effect on the quality of life as well as indirect positive effect on the life satisfaction.

Table 3. Model Fit Indices

\begin{tabular}{lll}
\hline Fit measures for SEM & Values recommended & Fit measure value \\
\hline Chi-square & $.05<$ & 308.976 \\
RMR & $.05>$ & .042 \\
RMSEA & $.1>$ & .062 \\
GFI & $.9<$ & .958 \\
AGFI & $.9<$ & .924 \\
NFI & $.9<$ & .908 \\
IFI & $.9<$ & .932 \\
CFI & $.9<$ & .930 \\
TLI & $.9<$ & .911 \\
\hline RFI & $.9<$ & .902 \\
\hline
\end{tabular}

Table 3 shows model fit indices. The values of all indices are compared with the threshold values. The value of chi- square is greater than the.o5 which show that the model fit is significant and acceptable.

The value of chi square is acceptable according to the assumption of multivariate normal distribution. The move towards further measurement of the model fit indices is needed because chi-square is not sufficient for defining all hypotheses. As per the Kelloway (1998) study, to accept or reject the null hypothesis, the result of chi square is not sufficient. In addition to absolute values, which are the adjusted goodness-of-fit index (AGFI), and the goodness-of-fit index (GFI), the root mean squared error of approximation (RMSEA), the root mean squared residual (RMR), NFI, CFI and the IFI as comparative fit measures were also examined. The value of GFI and AGFI ranges from 0-1 and the value of this is above. 9 which indicates good fit of the model. The value of Normed Fit Index (NFI), Comparative Fit Index (CFI), Tucker Louis Index (TLI) and incremental fit index (IFI) also ranges from 0 to 1 and the value. 9 indicates a good fit model. Root mean square residual is less than.05 and value of root mean square error of approximation is also lesser than the value of.1; these values show good fit of the model. 


\subsection{Results of Hypothesis Testing}

Family support and friends support both have positive significant effect on the quality of life of the retired person. Thus, hypothesis one is accepted. Therefore, hypothesis two is accepted. Quality of life is having significant positive effect on the life satisfaction. Thus, hypothesis three is accepted.

\section{Discussions}

The present study establishes relationships between social support, quality of life and life satisfaction. Findings of the study reveal that social support has significant impact on the quality of life of retired persons. Perceived social support from family has significant effect on the quality of life of retired person. After retirement, support from family plays significant role because retirement is not an individual phenomenon. It is a significant event for the whole family. After retirement, the daily routine of retired person changes after retirement. Retired people want to spend time with family, in order to kill the loneliness. Spouse and other family members affect the quality of life of the person. After family people seek support from the friends. Thus, friends support also affects the quality of life of retired person. Present study establishes a positive relationship between friends support and quality of life which means that support from family and friends increases the quality of life of retired person in retirement years. Quality of life has significant effect on the life satisfaction. If people get support from family and friends than enjoys a life with quality and feels satisfied.

\section{References}

1. Andrews., F.A., \& Withey, S.B. (1976). Social indicators of well-being in America: The development and measurement of perceptual indicators. New York: Plenum Press.

2. Berg, A.I., Hoffman, L., Hassing, L.B., McClearn, G.E., \& Johansson, B. (2009). What matters, and what matters most, for change in life satisfaction in the oldest-old? A study over 6 years among individuals 80+. Aging and Mental Health, 13(2), 191-201.

3. Biegel, D.E., Shore, B.K., \& Gordon, E. (1984). Building support networks for the elderly. Sage Publications.

4. Bosworth, H.B., \& Schaie, K.W. (1997). The relationship of social environment, social networks, and health outcomes in the Seattle Longitudinal Study: Two analytical approaches. The Journals of Gerontology Series B: Psychological sciences and social sciences, 52(5), 197-205.

5. Bowling, A., \& Windsor, J. (2001). Towards the good life: A population survey of dimensions of quality of life. Journal of Happiness Studies, 2(1), 55-82.

6. Chadha, N.K., \& Van Willigen, J. (1995). The Life scale: The development of a measure of successful aging. Indian Journal of Gerontology, 9(3), 83-90.

7. Obb, S. (1976). Social support as a moderator of life stress. Psychosomatic Medicine 38, 300-314.

8. Cohen, S.E., \& Syme, S.L. (1985). Social support and health. Academic Press.

9. Cummins, R.A., (1997). Assessing quality of life. Quality of life for people with disabilities: Models, research and practice, $2,116-150$.

10. Diener, E., Emmons, A., Larsen, R.J., \& Griffin, S. (1985). The satisfaction with life scale. Journal of Personality Assessment, 49(1), 71-74.

11. Farquhar, M. (1995). Definitions of quality of life: a taxonomy. Journal of advanced nursing, 22(3), 502-508.

12. Fitzpatrick, R. (1996). Quality of Life and Health: Concepts, Methods and Applications. Health Policy, 37(1), 53-72.

13. Furmonavicius, T. (2003). Impact or physiological factors on some dimensions of health-related quality of life of elder men. Medicina, 39(9), 896-901.

14. Gilgeous, V. (1998). Manufacturing managers: their quality of working life. Integrated Manufacturing Systems, 9(3), 173-181.

15. Jamuna, D., \& Ramamurthi, P.V. (1988). A study of Life Satisfaction in relation to I-E Locus of Control among Middle Aged and Older Women. Journal of Personality and Group Behavioul, 8(1), 1-6.

16. Kaur, H., Kaur, H., \& Venkateashan, M. (2015). Factors determining family support and quality of life of elderly population. International journal of medical science and public health, 4(8), 1049.

17. Lena, A., Ashok, K., Padma, M., Kamath, V., \& Kamath, A. (2009). Health and social problems of the elderly: A cross-sectional study in Udupi Taluk, Karnataka. Indian journal of community medicine: official publication of Indian Association of Preventive \& Social Medicine, 34(2), 131.

18. McCall S., (1975). Quality of Life, Social Indicators Research 2, 229-248. 
19. Naing, M.M., Nanthamongkolchai, S., \& Munsawaengsub, C. (2010). Quality of life of the elderly people in einme township irrawaddy division, Myanmar. Asia journal of public health, 1(2), 4-10.

20. kumagba, P.O. (2011). Family support for the elderly in delta state of Nigeria. Studies on Home and Community Science, 5(1), 21-27.

21. Phillips, D. (2006). Quality of life and sustainability. International Journal of Environmental, Cultural, Economic and Social Sustainability, 2(2), 103-112.

22. Pilisuk, M., \& Parks, S.H. (1981). The place of network analysis in the study of supportive social associations. Basic and Applied Social Psychology, 2(2), 121-135.

23. Ramamurthy P.V., \& Jamuna D. (1991). Social Supports Inventory for the Elderly. Journal Psychological Researches, 35, 133- 1136.

24. Ramamurthy P.V., \& Jamuna D. (1993). Psychological Dimensions of Ageing in India. Indian Journal of Social Sciences, 6(4), 309-331.

25. Rawlins, W. (2017). Friendship matters. Routledge.

26. Retirement, H. (2017). Issues Faced by Aging \& Retired Seniors. Retrieved from https://www.holidayretirement.com/aging-retirement-issues/.

27. Shanas, E. (1979). The family as a social support system in old age. The gerontologist, 19(2), 169-174.

28. Shin, D.C. (1979). The Concept of Quality of Life and the Evaluation of Developmental Effort: Some Applications to South Korea. Comparative politics, 11(3), 299-318.

29. Suber, P. (1996). Against the Sanctity of Life.

30. Szinovacz, M.E. (2003). Contexts and pathways: Retirement as institution, process, and experience. Retirement: Reasons, processes, and results, 6-52, New York: Springer.

31. Uchino, B.N. (2004). Social support and physical health: Understanding the health consequences of relationships. Yale University Press.

32. Uchino, B.N. (2004). Social support and physical health: Understanding the health consequences of relationships. Yale University Press.

33. Unsar, S., Erol, O., \& Sut, N. (2016). Social support and quality of life among older adults. International Journal of Caring Sciences, 9(1), 249-257.

34. Whoqol Group. (1995). The World Health Organization quality of life assessment (WHOQOL): position paper from the World Health Organization. Social science \& medicine, 41(10), 1403-1409.

35. Yuh, J., \& Choi, S. (2017). Sources of social support, job satisfaction, and quality of life among childcare teachers. The Social Science Journal, 54(4), 450-457.

36. Zimet, G.D., Dahlem, N.W., Zimet, S.G., \& Farley, G.K. (1988). The multidimensional scale of perceived social support. Journal of personality assessment, 52(1), 30-41. 